\title{
Community Involvement in Development of Evidence-Informed Recommendations for Rehabilitation for Older Adults Living with HIV
}

Solomon P., O’Brien K.K., Baxter L., MacLachlan D., Robinson G.

Version Final/Publisher Version

Citation Solomon P, O’Brien KK, Baxter L, MacLachlan D, Robinson G.

(published version) Community Involvement in Development of Evidence-Informed

Recommendations for Rehabilitation for Older Adults Living with HIV.

Progress in Community Health Partnerships: Research, Education and

Action. Volume 10, Issue 1, Spring 2016, pp. 83-8. DOI:

10.1353/cpr.2016.0008

Publisher's Statement Copyright (C) 2016 The John Hopkins University Press. This article first appeared in Progress in Community Health Partnerships: Research, Education and Action, Volume 10, Issue 1, Spring, 2016, pages 83-8.

How to cite TSpace items

Always cite the published version, so the author(s) will receive recognition through services that track citation counts, e.g. Scopus. If you need to cite the page number of the TSpace version (original manuscript or accepted manuscript) because you cannot access the published version, then cite the TSpace version in addition to the published version using the permanent URI (handle) found on the record page. 


\section{PROJECT MUSE'}

Community Involvement in Development of Evidence-Informed Recommendations for Rehabilitation for Older Adults Living With HIV

Patricia Solomon, Kelly K. O’Brien, Larry Baxter, Duncan MacLachlan, Greg Robinson

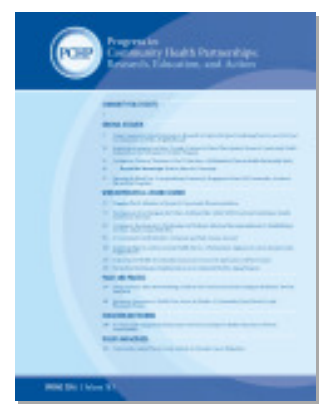

Progress in Community Health Partnerships: Research, Education, and Action, Volume 10, Issue 1, Spring 2016, pp. 83-88 (Article)

Published by Johns Hopkins University Press

DOI: $10.1353 /$ cpr.2016.0008

$\Rightarrow$ For additional information about this article https://muse.jhu.edu/article/612664 


\title{
Community Involvement in Development of Evidence-Informed Recommendations for Rehabilitation for Older Adults Living With HIV
}

\author{
Patricia Solomon, PhD ${ }^{1}$, Kelly K. O'Brien, PhD ${ }^{1,2}$, Larry Baxter ${ }^{3}$, Duncan MacLachlan ${ }^{4}$, Greg Robinson ${ }^{3}$ \\ (1) School of Rehabilitation Science, McMaster University; (2) Department of Physical Therapy, University of Toronto; (3) Canadian Working Group on HIV and Rehabilitation; \\ (4) AIDS Committee of Toronto \\ Submitted 25 August 2014; Revised 07 January 2015; Accepted 23 January 2015.
}

\section{Abstract}

Background: Involving community in development of clinical practice guidelines (CPGs) can decrease the gap between patient preferences and research evidence.

Objective: To incorporate meaningful participation of people living with human immunodeficiency virus (HIV; people living with HIV [PHAs]) in the development of evidence informed recommendations for rehabilitation practice.

Methods: PHAs were involved in a process to develop practice recommendations internally as members of a project team and externally through formal endorsement of the recommendations.

Lessons Learned: Lessons learned include 1) providing time to develop as a team and understand the roles, biases, and expertise of each member, 2) engaging community in initial discussions to determine the most meaningful involvement, 3) realizing that participation in research may trigger anxiety and stress in community members, 4) developing terms of reference to clarify roles and expectations, 5) providing opportunities for skill development, and 6) conducting formal evaluation of the process and satisfaction of community.

Conclusion: Meaningful inclusion of community can improve the quality of practice guidelines.

\section{Keywords}

Community-based participatory research, community health partnerships, power sharing, process issues, rehabilitation centers, health care quality, access, and evaluation

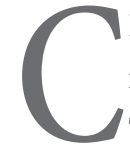

PGs have become increasingly popular and necessary for clinicians to engage in evidence based practice. The importance of incorporating a community perspective that reflects patient or consumer values and preferences in guidelines has been emphasized in recent years as a means to decrease the gap between patient preferences and best evidence and ultimately improve quality of care. ${ }^{1}$ The growing literature in this area suggests that patient involvement should be at all stages of guideline development ${ }^{2}$ and highlights the importance of training patients for their role. ${ }^{3}$ However, inclusion of patients or consumers in guideline development is not without controversy. Critics state that patient involvement could make developing guidelines more expensive and result in guidelines that are more complex hindering translation into practice. ${ }^{4,5}$ Others argue for reconsideration of involvement of patients in the process as evidence to support improvement in the quality of the guidelines through patient participation does not exist. ${ }^{6}$

Other concerns relate to the perceived meaningfulness and importance of patient contributions. In their knowledge synthesis, Legare et al. ${ }^{1}$ note that there is often a distinction between the technical, knowledge based aspects of the guideline development assumed by the researchers, and the values and preferences aspects assumed by the patients. Some suggest 
that patient involvement be more targeted and used at strategic decision points. ${ }^{7}$ It is not only a matter of when in the process that patient involvement would be most meaningful; there is the form of the involvement or how the patient is involved.

Decisions of when and how patients should be involved in guideline development are complex and depend on the content area. Patient participation may be most important in areas where there is little or no evidence. ${ }^{3}$ Rehabilitation in the context of HIV is an evolving area of practice as HIV transitions from an acute fatal illness to a chronic illness in which people living with HIV with access to medications live longer with episodic periods of wellness and illness. This increased longevity is accompanied by common comorbidities related to aging, the virus, and antiretroviral medications.

Rehabilitation can be defined broadly as any services or providers with the aim to reduce impairments, activity limitations or social participation restrictions experienced by an individual. ${ }^{8}$ As with many emerging areas of practice, developing evidence-based guidelines related to HIV and rehabilitation is challenging because high levels of evidence, such as systematic reviews and meta-analyses on effectiveness of interventions are largely absent. In previous work, we conducted interviews and focus groups with 28 stakeholders in the field of HIV and rehabilitation and developed recommendations for the process of developing CPGs in this area. ${ }^{9}$ Recommendations stated that HIV rehabilitation practice guidelines should be flexible, strike a balance between a broad and a specific scope, take on a variety of formats for different end users, be interprofessional, and have clear strategies for knowledge translation. ${ }^{9}$ Additionally, recognizing the lack of high-quality research, stakeholders suggested that the term "evidence-informed recommendations" would be preferable to CPGs.

\section{Objectives}

We developed evidence-informed recommendations for rehabilitation with older adults aging with HIV using a community-engaged approach. ${ }^{10}$ In Canada, there is a long history of HIV community involvement in research. This is evidenced by the Canadian Institutes for Health Research funding of an HIV/AIDS Community-Based Research Program ${ }^{11}$ and the establishment of the Canadian Institutes for Health Research HIV/AIDS Research Advisory Committee that includes community representation in its priority setting processes. ${ }^{12}$ As proponents of the Meaningful Involvement of People Living with HIV Principle, our goal was to ensure meaningful participation of people living with HIV in the development of the recommendations. ${ }^{13}$ In this paper, we describe the process by which we incorporated HIV community involvement into the methodology of recommendations development. As is the tradition in HIV-related research, we refer to our HIV community members as people living with HIV (PHAs) throughout the paper. This project was conducted in partnership with the Canadian Working Group on HIV and Rehabilitation $\left(\mathrm{CWGHR}^{14}\right)$ a national multisector charitable organization that promotes innovation and excellence in rehabilitation in the context of HIV.

\section{METHODS}

Our goal was to develop evidence-informed recommendation on rehabilitation care, treatment, and support for older adults aging with HIV. We conducted a knowledge synthesis of two bodies of research evidence: 1) research specific to nonpharmacologic rehabilitation interventions and management of PHAs and 2) high-level evidence on rehabilitation interventions for common comorbidities identified in older adults with HIV. This project was approved by the Research Ethics Board at McMaster University in Hamilton, Canada.

Two types of community (PHA) involvement were included: internally as key members of the project team and externally through endorsement of the recommendations. PHA involvement began with the initial proposal development. The project team was interdisciplinary and consisted of three adults living with HIV, five clinicians, five researcher/ educators, and two policy stakeholders with expertise in HIV, aging, and rehabilitation. The team provided oversight to the entire process; thus, the values and preferences of all team members were incorporated into the recommendations throughout. Over the 3-year period, the research team met twice by teleconference and three times face to face to make decisions during all steps of the knowledge synthesis process and to draft the recommendations. Participants in the process were reimbursed for travel costs only.

The synthesis was a complex iterative process occurring over three phases. Phase 1 commenced with the identification of articles through a literature search and determining inclusion eligibility. The PHAs on the team provided input 
into the search terms and strategies. For example, although not initially identified as a common comorbidity one PHA had direct exposure to several individuals who were living with HIV and Parkinson's disease and felt that it should be included as part of the search strategy. The search yielded 6,664 independent citations. Specific details on the knowledge synthesis procedure and the detailed recommendations are available elsewhere. ${ }^{10}$

The next steps included screening the abstracts for inclusion, data abstraction, grading of methodological quality, data synthesis, and development of preliminary recommendations. Given that the data abstraction and synthesis component of the knowledge synthesis process is typically time consuming, involving extensive appraisal of the literature, the team discussed the feasibility of PHA involvement at this stage in the development of the recommendations. Each PHA team member determined the extent to which he could be involved in this phase. Ultimately, one of three PHAs was involved, working in conjunction with the researchers and staff to conduct this component of the process. The end result was 74 preliminary recommendations synthesized from 165 articles. Each team member then provided a GRADE rating (Grading of Recommendations Assessment, Development and Evaluation), which incorporated both the quality of the evidence and the extent to which the recommendation related to older adults living with HIV. ${ }^{10}$ This process required the team members to consider the balance between the benefits and shortcomings of each recommendation with the values of the researchers, clinicians, and PHA team members. Team members provided comments on their preferences and suggested revisions to the recommendations. Decisions were made by consensus at team meetings.

In Phase 2, the team reviewed the GRADE results and preliminary recommendations, incorporating values and preferences of all team members, including PHAs, into the revisions. This involved the consideration of both the quality of the evidence and the extent to which the individual felt the recommendation related to older adults living with HIV. Each team member was asked to comment on his/her values and preferences and how these influenced their ratings. Raters had to determine, in the absence of high level evidence, whether they would endorse a specific recommendation. After the rating exercise, the team met to debate, revise, and consolidate the recommendations. Final decisions and wording of the recommendations were accomplished through consensus. The end result was 54 evidence-informed recommendations ready for external endorsement.

Phase 3 involved extensive consultation with community members external to the project team. The project team developed a list of 38 key informants who were personal contacts with expertise in HIV, people living with HIV, and/ or members of the CWGHR. Key informants were sent an email invitation to participate in the endorsement of the preliminary recommendations. Nineteen individuals responded to the invitation and completed an online endorsement survey (nine health professionals, eight PHAs, and two who were both PHAs and health professionals). Participants were sent each recommendation with the level of evidence from which it was derived and access to the citation and asked whether they endorsed, did not endorse or had no opinion about each recommendation. This was incorporated into the final recommendations of 52 specific and 8 overarching evidence-informed recommendations for rehabilitation with older adults with HIV.

\section{DISCUSSION}

There is a need to make research processes more accessible to non-researchers to promote meaningful participation of community in guideline development. Our process of incorporating the PHAs' values and preferences was unique in several ways. The background work to establish guiding principles for development of recommendations involved extensive consultations with all stakeholders, including PHAs, and provided the foundation for this work. ${ }^{9}$ The principles emphasized understanding the diversity of PHAs, their multiple vulnerabilities and sources of stigma and emphasized a patient centered approach to guideline development. Our process promoted the guiding principles through involvement of the HIV community in two ways: internally through being a member of the investigative team and externally through a formal external endorsement process. We developed recommendations in an area in which there was very little high level research evidence thus placing additional importance on the experiences, preferences and values of the community members. Krahn and $\mathrm{Naglie}^{2}$ note the need to integrate "preference related evidence" into recommendations through distinguish- 
ing between recommendations that nearly all patients will accept and those that may vary depending on patient choice. This was a necessity in our process given the low levels of evidence; we believe this was a strength of our approach. We offer the following lessons learned from our experiences to be considered by researchers working with community members to develop evidence-informed recommendations.

1. Provide time to develop as a team and to discuss and understand the roles, perspectives, biases, and expertise of each member. Although logistically many of the meetings were held by teleconference, face-to-face meetings were important to establish a sense of team connectivity and to promote robust discussion. Team members were mindful of their role in providing their perspectives and values and prompted considerable debate throughout the process. The team should discuss at the beginning of the process and revisit throughout whether the community member is expected to represent a constituency and/or bring personal experience to the table. ${ }^{15}$ The PHAs on our team had prior research experience and knowledge of HIV and rehabilitation and hence also contributed to the methodological discussions. Although we believe this was a strength, others argue that highly experienced community representatives become fellow academics and thus are not representative of the general community perspective and experiences. ${ }^{6}$

2. Engage PHAs in initial research discussions to determine how and when involvement would be most meaningful and comfortable. The development of evidence-informed recommendations is a comprehensive and often onerous task. Early discussions among our team determined that it would be most efficient for research staff to do the initial data extraction and critical appraisal of the articles, tasks that did not necessarily require the knowledge related to patient values and preferences that PHAs on the team could provide. Although some advocate participation of patients through all phases of guideline development this may not be practical, feasible or affordable. ${ }^{6}$ Intensive community participation in guideline development can be costly requiring significant time investment from both researchers and community members. ${ }^{6}$ One challenge for community members on our team related to other commitments in the form of employment or otherwise, which meant they had limited time to devote their expertise to this project. This was compounded by the complexities of the project which extended our deadlines and resulted in a longer time commitment than expected. Nevertheless, our process of community input from both internal team members and through external endorsement is a way to include diverse perspectives without resorting to a large internal team, which may increase costs and complexity and hamper decision making. ${ }^{16}$

3. Be mindful that engaging in research may trigger stress and anxiety among community members. In our team, the activity of reviewing a vast amount of literature on comorbidities, disability, and aging with HIV was raised as anxiety provoking for PHAs who themselves were aging with HIV. This highlights the importance of carefully considering the nature and extent of community engagement when it comes to guideline development, the potential risks of involvement and the need to provide support for dealing with discomforting findings. In this situation, it is critical to incorporate strategies to provide support to empower PHAs to remain engaged in research. While we were unable to find other literature that refers to anxiety created through involvement in the guideline development process our experiences highlight the need for researchers to be mindful of this possibility especially in vulnerable populations. Aging with HIV is a relatively recent phenomenon fraught with uncertainty, ${ }^{17}$ which may highlight vulnerabilities in this population.

4. Because it is difficult to anticipate areas in which there may be controversy in research and, specifically, development of practice recommendations, we recommend developing terms of reference and decision-making processes for all teams, including those with previous experience working together. In our study, PHAs on the team were knowledgeable and well-versed in HIV and rehabilitation research and had a history of working with the researchers. We did not experience process-related issues; differences of opinion were resolved by consensus. However, challenges could arise if community members have varying levels of expertise and opposing views when trying to decide on inclusion of evidence, or the exclusion of certain recommendations. Developing terms of reference can help to formalize the structure of partnership and clarify roles and expectations among researchers and community members. ${ }^{18}$ Although we did not develop terms of reference or a formalized process for resolving conflict, we believe this is important in early stages of team formation. Pagliari et al. ${ }^{16}$ go further by suggesting that methods used to achieve consensus should be reported by guideline developers to allow users to assess the potential influence of group process on the validity of the decision-making process. 
5. Allow opportunities for training and mentoring for those who want to develop skills in research. As noted, our team benefited through the contributions of PHAs to all components of the research in addition to providing their personal values and preferences throughout the process. Although the literature often makes a distinction between the methodological expertise provided by the researchers and patients' values and preferences, we do not see these as discreet entities. We believe that, to best reflect Meaningful Involvement of People Living with HIV, researchers need to be open to varied contributions and provide ongoing opportunities and support for skill development. Capacity building can also occur in academic researchers. In our instance, researchers and PHAs on the team had the opportunity to develop collaborations over the years through the leadership of CWGHR. This resulted in a strong foundational partnership enabling us to successfully develop the recommendations. This relationship has been mutually beneficial, allowing academic researchers and the community to learn how to effectively work together in a complex and multi-staged process of evidence-informed recommendations development.

6. Build in a formal evaluation of the recommendation (or guideline) development process and the PHAs' level of satisfaction with their involvement. We support calls for more rigorous evaluation of the "value added" of community involvement in guideline development. ${ }^{16}$ The goal of incorporating patients' preferences in the guideline development process varies considerably. Boivin et al. ${ }^{19}$ note that there are fundamental differences between social perspectives that seek to maximize population health benefits, those that promote individual tailored approaches, and those that promote involvement for political legitimacy. Future work needs to be clear about the rationale for community participation to ensure that the appropriate outcome measures are chosen to evaluate the effectiveness of patient their involvement.

It is important to note that involvement of the PHAs was also a form of integrated knowledge translation to help ensure that the recommendations are most relevant and applicable to all relevant stakeholders and knowledge users. The involvement of the CWGHR as a key knowledge user on the project was an important strategy to ensure translation to clinicians, PHAs, and community organizations. Next steps include the development of fact sheets targeted to these groups to translate the recommendations into practice. Although not part of this project, PHAs will remain integrally involved and take a leadership role in this process.

These lessons build on the work of others who promote the use of more than one method of patient involvement in guideline development. ${ }^{3}$ We believe these lessons learned and subsequent recommendations are of broad interest to all researchers wishing to incorporate community perspectives meaningfully into guideline development.

\section{CONCLUSIONS}

We provide recommendations to increase the meaningful involvement of community in the development of evidence-informed guidelines. Benefits of including PHAs in guideline development go beyond increasing the quality of the guidelines. In vulnerable and stigmatized populations involving community can foster empowerment and promote legitimacy and partnerships between clinicians, academics and researchers. Involving PHAs internally as members of the core research team, and as part of the external endorsement process when developing evidence-informed recommendations, provides a process to include community in the development of high-quality evidence that can help to enhance HIV care and practice.

\section{ACKNOWLEDGMENTS}

This research was supported by a grant from the Canadian Institutes of Health Research. Kelly O'Brien is supported by a Canadian Institutes of Health Research New Investigator Award. 
1. Legare F, Boivin A, van der Weijden T, Pakenham C, Burgers J, Legare J, et al. Patient and public involvement in clinical practice guideline: A knowledge synthesis of existing programs. Med Decis Making. 2011;31:E45-E74.

2. Krahn M, Naglie G. The next step in guideline development: incorporating patient preferences. JAMA. 2008;300(4):436-8.

3. Van Wersch A, Eccles M. Involvement of consumers in the development of evidence based clinical guideline: Practical experiences from the North of England evidence based guideline development program. Quality in Health Care. 2001;10:10-6.

4. Umscheid C. Should guidelines incorporate evidence of patient preferences? J Gen Intern Med. 2009;24(8):988-90.

5. Van der Weijden T, Legare F, Boivin A, Burgers J, van Veenendaal H, Stiggelbout A, et al. How to integrate individual patient values and preferences in clinical practice guidelines? A research protocol. Implement Sci. 2010;5:10.

6. Van de Bovenkamp HM, Trappenburg M. Reconsidering patient participation in guideline development. Health Care Anal. 2009;17:198-216.

7. Rogers WA. Evidence-based medicine in practice: Limiting or facilitating patient choice? Health Expectations. 2002;5:95-103.

8. Worthington C, Myers T, O'Brien K, Nixon S, Cockerill R, Bereket, T. Rehabilitation professionals and human immunodeficiency virus care: results of a national Canadian survey. Arch Phys Med Rehabil. 2008;89(1):105-13.

9. O'Brien K, Wilkins A, Zack E, Solomon P. Developing clinical practice guidelines in HIV rehabilitation: Process recommendations and guiding principles. AIDS Educ Prev. 2011;23(5): 457-68.

10. O'Brien KK, Solomon P, Trentham B, MacLachlan D, MacDermid J, Tynan AM, et al. Evidence-informed recommendations for rehabilitation with older adults living with HIV: A knowledge synthesis. BMJ Open. 2014;4(5):e004692.
11. Canadian Institutes of Health Research. CIHR HIV/AIDS Community-Based Research Program [cited 2014 Dec 15]. Available from: www.cihr-irsc.gc.ca/e/25835.html

12. Canadian Institutes of Health Research (CIHR). CIHR HIV/ AIDS Research Advisory Committee [cited 2014 Dec 15]. Available from: www.cihr-irsc.gc.ca/e/25834.html

13. Global Network of People Living with HIV/AIDS (GNP+) and the Asia Pacific Network of People Living with HIV/AIDS $(\mathrm{APN}+)$. Self-assessment checklist: Meaningful involvement of PLHIV and affected communities (MIPA). NGO Code of Good Practice [cited 2014 Aug 11]. Available from http:// hivcode.org/silo/files/final-mipa-.pdf

14. Canadian Working group on HIV and Rehabilitation (CWGHR) [cited 2015 Jan 5]. Available from: hivandrehab.ca

15. Boivin A, Currie K, Fervers B, Gracia J, James M, Marshall C, et al. Patient and public involvement in clinical guidelines: international experiences and future perspectives. Qual Saf Health Care. 2010;19:e22

16. Pagliari C, Grimshaw J, Eccles M. The potential influence of small group processes on guideline development. J Eval Clin Prac. 2001;7(2):165-73.

17. Solomon P, O'Brien KK, Wilkins, S, Gervais N. Aging with HIV and disability: the role of uncertainty. AIDS Care. 2014;26(2):240-5.

18. Sadler LS, Larson J, Bourgey S, Lapaglia, Bridger L, McCaslin C, et al. Community-university partnership in community-based research. Prog Community Health Partnersh. 2012;6(4):463-9.

19. Boivin A, Green J, van der Meulen J, Legare F, Nolte E. Why consider patients' preferences? A discourse analysis of clinical practice guideline developers. Med Care. 2009;47(8):908-15. 